\title{
Herniorrafias umbilicales con cloruro de polivinilo (PVC) en cerdos (sus scrofa domesticus): estudio piloto
}

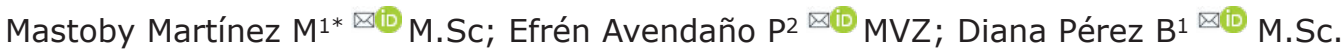

\begin{abstract}
${ }^{1}$ Universidad de Córdoba, Facultad de Medicina Veterinaria y Zootecnia, Departamento de Ciencias Pecuarias, Grupo de Investigación en Medicina y Cirugía Veterinaria (MECIVET). Montería, Colombia.

²Ejercicio particular. Grupo de Investigación en Medicina y Cirugía Veterinaria (MECIVET). Montería, Colombia.

*Correspondencia: mmiguelmartinez@correo.unicordoba.edu.co
\end{abstract}

Recibido: Enero 2020; Aceptado: Febrero 2021; Publicado: Mayo 2021.

\section{RESUMEN}

Objetivo. Evaluar clínicamente la efectividad del PVC en herniorrafias umbilicales en cerdos. Materiales y métodos. Se utilizaron 16 cerdos clínicamente sanos, de ambos sexos, con edades entre dos y tres meses, de las razas Landrace y Landrace $x$ Pietran y peso promedio de 20 kilogramos. La inclusión se basó en el diagnóstico clínico de hernia umbilical. A todos se les implantó PVC como material protésico entre peritoneo parietal y músculos abdominales. Para la fijación de la bolsa se utilizó poliamida de 20 libras de presión con puntos en "U" (cuatro en total), quedando los nudos a dos centímetros del anillo y evitando con la tracción la formación de pliegues invertidos. El diámetro de la bolsa de PVC dependió del diámetro del anillo herniario, pero con la constante que fuese redondeada y cuatro centímetros mayores. Parámetros como temperatura, edema, color y dehiscencia de la herida se evaluaron por 15 días, mientras que recidiva de la hernia, fistulación y apetito se evaluaron por dos meses. Resultados. Se obtuvo un promedio de temperatura local de $37.5^{\circ} \mathrm{C}$. Halos tumorales con medidas de un centímetro a las 24 horas post-quirúrgico con involución hacia el final de la evaluación. El 100\% de los animales cicatrizó por primera intención. El 87.5\% no presentó recidiva de la hernia. El $100 \%$ no presentó signos compatibles con fístula o granuloma y el apetito fue normal. Conclusiones. Durante el periodo de evaluación, el PVC demostró ser efectivo en la corrección de hernias umbilicales en cerdos.

Palabras clave: Biomaterial; cloruro de polivinilo; hernia; umbilical (Fuente: MeSH).

\section{ABSTRACT}

Objective. To evaluate the clinical effectiveness of PVC on umbilical herniorrhaphy in pigs. Materials and methods. We used 16 clinically healthy pigs of both sexes, aged between 2 and 3 months, Landrace and Landrace $x$ Pietran breeds with an average weight of $20 \mathrm{~kg}$. Inclusion criteria were based on the clinical diagnosis of umbilical hernia. Herniorrhaphy was carried using PVC as a prosthetic material. The implantation of the material was made between parietal peritoneum and abdominal muscles by creating a pocket in $360^{\circ}$, with a depth of five centimeters. For the fixation of the bag, 35

Como citar (Vancouver)

Martínez MM, Avendaño PE, Pérez BD. Herniorrafias umbilicales con cloruro de polivinilo (PVC) en cerdos (sus scrofa domesticus): estudio piloto. Rev MVZ Córdoba. 2021; 26(2):e1867. https://doi.org/10.21897/rmvz.1867 
pounds of pressure polyamide was used with " $U$ " points in the fascio-muscular package (four stitches overall), the knots were two centimeters next to the ring, avoiding inverted edges with sustained traction. The size of the PVC bag depended on the size of the ring, but it was constantly rounded and four centimeters larger than the edge of the ring. The parameters evaluated for 15 days were temperature, edema, wound color and dehiscence, and relapse, fistula and appetite for 2 months. Results. An average local temperature of $37.5^{\circ} \mathrm{C}$ was obtained. Tumor halos of one centimeter at 24 hours post-surgery with involution towards the end of the evaluation. $100 \%$ of the animals healed by first intention. $87.5 \%$ did not present recurrence of the hernia. $100 \%$ did not present signs compatible with fistula or granuloma. Appetite was normal. Conclusions. During the evaluation period, PVC proved to be effective in the correction of umbilical hernias in pigs.

Keywords: Biomaterial; polyvinyl chloride; hernia; umbilical (Source: MeSH).

\section{INTRODUCCIÓN}

La cirugía en cerdo como paciente no siempre resulta positiva desde el punto de vista beneficio/ costo. Algunas condiciones frecuentes como hernia, prolapso, distocia y atresia pueden ser antieconómicas para la industria (1), estimándose el costo de materiales y del servicio profesional especializado para corregir una hernia umbilical en cerdo con promedio de $20 \mathrm{~kg}$ en alrededor de los 100 dólares (nota de autor).

Las hernias umbilicales están dentro de las patologías de frecuente presentación en cerdos con causas variadas como: genética, trastornos congénitos, infecciones umbilicales, debilidad tisular y manejo animal. De acuerdo al estudio sobre malformaciones congénitas en esta especie, se encontró que las hernias inguinoescrotales y las hernias umbilicales representan el $12.9 \%$ y el $45.2 \%$ de las patologías congénitas respectivamente (2).

La hernia umbilical es una de las variantes de las hernias ventrales en animales domésticos y se caracteriza por la salida de vísceras de la cavidad peritoneal a través del anillo, siendo el intestino delgado su principal contenido. Su diagnóstico es clínico, donde en la mayoría de los casos la palpación favorece la reintroducción del contenido herniario (hernias reducibles). Existe un pequeño porcentaje (hernias no reducibles) donde se recurre a las ayudas diagnósticas como la ecografía y/o radiología para su confirmación; siendo poco utilizadas en cerdos por razones de costos (3).

Aunque existen diversas técnicas quirúrgicas para la corrección de las hernias, no hay una que sea aceptada como la mejor a nivel mundial por los cirujanos, por esto, siempre se buscan nuevos métodos que cumplan con todas las expectativas a la hora de practicar una herniorrafia (3).
Las técnicas de corrección se clasifican en técnicas con tensión y técnicas sin tensión. Estas últimas se caracterizan por el empleo de mallas de diversos materiales que pueden ser no absorbibles o mixtas; sintéticas o naturales (fascia y membranas biológicas), que son implantadas sobre la aponeurosis abdominal, entre la aponeurosis y los músculos abdominales, entre los músculos abdominales y el peritoneo parietal o a nivel intra-peritoneal (3).

En cuanto a los biomateriales deben reunir una serie de condiciones como ser de fácil consecución, económicos, fáciles de esterilizar, que no requieran de instrumental especializado, no carcinogénicos, no alergénicos y sobre todo ser biotolerados en el local de implantación (3). EI PVC cuenta con características importantes como material protésico como lo son: posibilidad de esterilización, es inerte, fácil de manipular, su disponibilidad y su bajo costo (4).

EI PVC es un polímero derivado del cloruro de vinilo, de los más usados gracias a sus propiedades inherentes como resistencia a la abrasión y al impacto, ligero, impermeable, química y biológicamente inerte, duradero y resistente al fuego, no arde salvo en caso de estar en presencia de llama continua. Mediante el uso de aditivos tales como estabilizantes, plastificantes u otros, la resina de PVC se transforma en un material rígido o flexible, que permite una gran variedad de aplicaciones en distintas áreas como construcción, embalaje, mobiliario, industria eléctrica, industria del automóvil, aplicaciones médicas, servicios, agricultura y otros (4). El objetivo de la investigación fue evaluar clínicamente la efectividad del PVC en herniorrafias umbilicales en cerdos de las razas Landraces y Landraces $x$ Pietran. 


\section{MATERIALES Y MÉTODOS}

Tipo de estudio. Descriptivo.

Tipo de muestreo y cálculo del tamaño de la muestra. Para el estudio se utilizó un muestreo de tipo no probalístico por intencionalidad, dado a que por razones técnicas y económicas no se disponía de un mayor número de animales.

Aspectos éticos. La metodología de este trabajo fue aprobada por el Comité de Ética de la Facultad de Medicina Veterinaria y Zootecnia de la Universidad de Córdoba según acta Nº 004 del 3 de septiembre de 2010, teniendo en cuenta las normas técnicas para el uso de animales para experimentación, enmarcado en el cumplimiento de la "Declaración Universal de los Derechos de los animales", de las "Normas Internacionales para la Investigación Biomédica con animales" establecidas por la ONU, La Ley 84 de Octubre de 1989 Ministerio de salud de Colombia y la Ley 1774 del 6 de enero 2016 del código penal de la república de Colombia.

Localización. La investigación se realizó en la clínica veterinaria Julio E. Cuervo de la Facultad de Medicina Veterinaria y Zootecnia de la Universidad de Córdoba, Colombia, localizada en el Campus universitario Berástegui en Ciénaga de Oro-Córdoba.

Pacientes. Fueron utilizados 16 cerdos de ambos sexos, con edades entre dos y tres meses, de las razas Landrace y Landrace $x$ Pietran y con peso promedio de $20 \mathrm{~kg}$. La inclusión se basó en el diagnóstico clínico de hernia umbilical. Durante el estudio fueron mantenidos en piaras con alimento concentrado dos veces al día y agua a voluntad. Los animales fueron proporcionados por el programa productivo porcino de la Facultad de Medicina Veterinaria y Zootecnia.

Métodos e instrumentos de recolección de datos. A los pacientes se les realizó un muestreo sanguíneo para tener idea del estado general. Durante el post-operatorio de 15 días, se evaluaron parámetros como, temperatura, edema y color de la región umbilical, dehiscencia de la herida, recidiva de la hernia, fistulación y apetito del animal. Para la interpretación de los datos recolectados se utilizó como medida estadística la línea de tendencias.

Material empleado. El PVC implantado se obtuvo de bolsas fabricadas para contener soluciones de fluido-terapia. La primera bolsa envoltorio se desechó, empleándose para la investigación exclusivamente la bolsa recipiente del líquido, que viene esterilizada de acuerdo al fabricante (Fresenius Kabi Colombia S.A.S).

Pre-quirúrgico. Los pacientes, fueron sometidos a ayuno sólido de 24 horas con acceso a agua permanente. En las zonas anatómicas involucradas en el procedimiento se realizó la rutina de antisepsia técnicamente recomendada con yodopovidona, con previo lavado con agua y jabón. Como protocolo anestésico se utilizó azaperona (3 $\mathrm{mg} / \mathrm{kg}$ de peso/im y propofol $(10 \mathrm{mg} / \mathrm{kg}$ de peso/iv) como pre-anestésico e inductor respectivamente. El mantenimiento anestésico se consiguió con isofluorano al 3\% (5). Para la aplicación de fármacos e hidratación se canalizó previamente la vena auricular marginal y se mantuvo un goteo de 20 gotas/ minuto de lactato de Ringer.

Descripción del abordaje quirúrgico. La técnica quirúrgica realizada se basó en la técnica de Rives-Stoppa quienes implantaron malla en el espacio pre-peritoneal (6), local escogido para colocar la bolsa de PVC y que se describe a continuación:

Se realizó incisión elíptica en la piel, que tuvo como centro al saco herniario externo, que se desbridó completamente (Figura 1A). Seguidamente, se hizo divulsión del tejido celular subcutáneo con dedo montado con gasa hasta la base del anillo herniario, lo que dejó libre al saco herniario interno que fue introducido a la cavidad abdominal digitalmente o con pinza de disección, lo que facilitó la identificación del anillo herniario (Figuras $1 \mathrm{~B}$ y $1 \mathrm{C}$ ). Inmediatamente se incidió en $360^{\circ}$ la fascia abdominal a un $\mathrm{mm}$ del borde del anillo herniario (Figura 1D) para desprendió con tijera de Mayo o digitalmente el saco herniario interno de su adherencia muscular, y se creó un bolsillo con fondo máximo de cinco centímetros independiente del diámetro del anillo (Figuras $2 \mathrm{~A}$ y $2 \mathrm{~B}$ ).

El tamaño de la bolsa de PVC a implantar dependió de la medida del anillo (Figuras $2 \mathrm{C}$ y 2D), la bolsa fue recortada en forma redondeada con un tamaño de cuatro centímetros mayor al diámetro del anillo. Se continuó con la colocación de cuatro puntos en " $U$ " en la bolsa a las 9:00, $3: 00,6: 00$ y $12: 00$, de acuerdo con las manecillas del reloj. La sutura utilizada fue poliamida de 20 lb de presión (Figura 3A). 


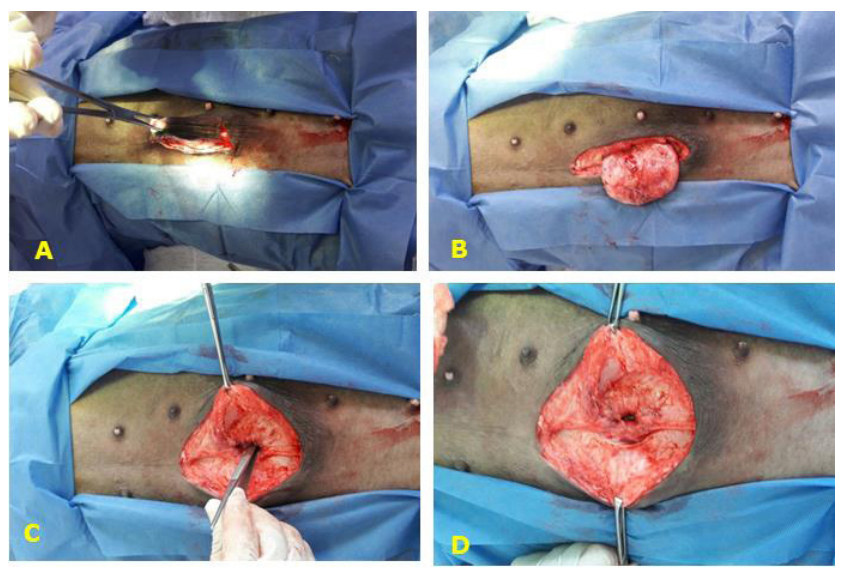

Figura 1. A) Incisión cutánea. B) Divulsión del saco herniario interno. C) Introducción del saco peritoneal e identificación de los bordes del anillo herniario. D) Incisión de la fascia abdominal a un $\mathrm{mm}$ del borde del anillo herniario.
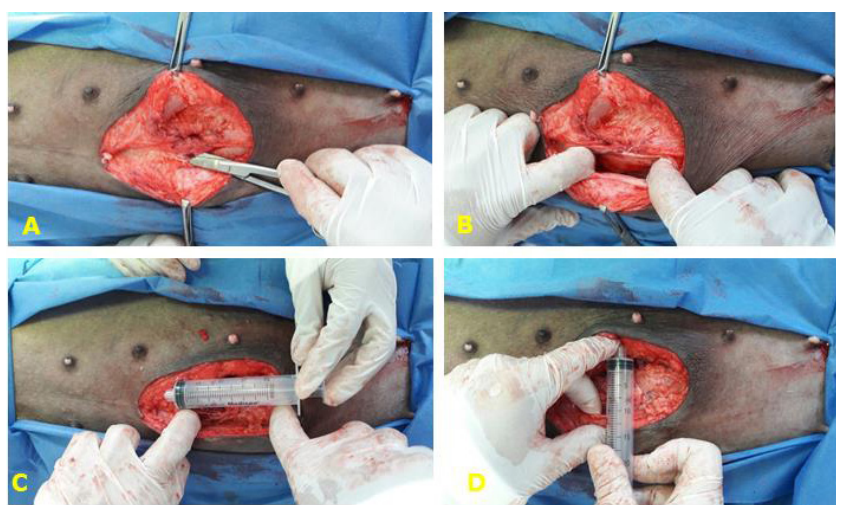

Figura 2. A) Inicio de la divulsión del saco peritoneal con tijera de Mayo. B) Continuación de la divulsión con los dedos. C) Medida longitudinal del anillo herniario. D) Medida transversal del anillo herniario.

Para facilitar el paso de los cantos de poliamida por la fascia abdominal y los músculos, los investigadores diseñaron una aguja con formato de "U" con un ojal en cada punta. Los dos primeros puntos fueron colocados en craneal y caudal del anillo (9:00 y 3:00) (Figura 3B), seguidos por los dos puntos laterales $(12: 00$ y 6:00). Los nudos quedaron a dos centímetros en relación al borde del anillo. Para evitar que la bolsa de PVC quedara con pliegues invertidos, se realizó tracción sostenida de los hilos y al tiempo se aprovechó para acomodar a los mismos (Figura 3C).

Una vez implantada la bolsa de PVC en el espacio pre-peritoneal, se suturó con poliglactín 910 del calibre 2-0 y punto simple continuo los bordes del pliegue fascio-musular, con tensión sostenida y ligera sin llegar al afrontamiento de sus bordes (Figura 3D). Este paso ayudó a reducir el espacio muerto. Los planos restantes se suturaron de manera rutinaria.

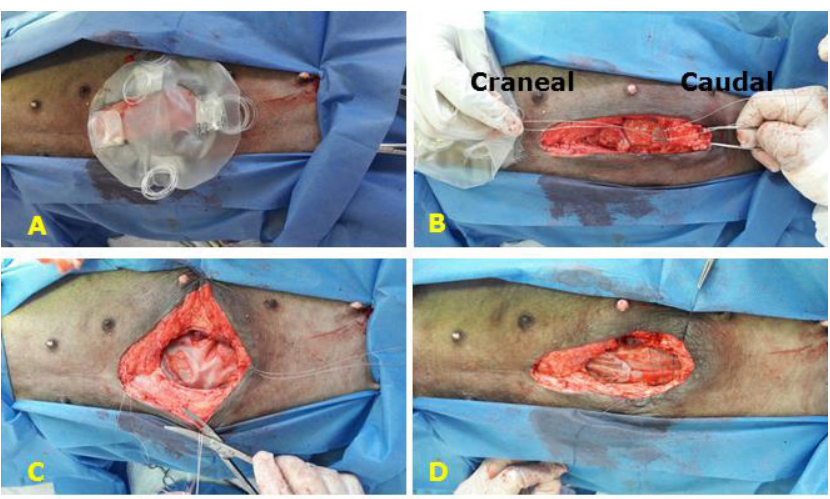

Figura 3. A) Moldeado de la bolsa de PVC y colocación de puntos en "U". B) Introducción de la aguja en "U" para el paso de los cantos del hilo. C) Sutura y corte de los cantos de poliamida. D) Bolsa de PVC implantada en el espacio pre-peritoneal.

Post-quirúrgico. Se utilizó como terapia antibiótica dosis única de oxitetraiclina L.A. a $20 \mathrm{mg} / \mathrm{kg}$ de peso vivo/im y como terapia antiinflamatoria betametasona en dosis de $2 \mathrm{mg} /$ $\mathrm{kg}$ de peso vivo/im/3 días. En el post-quirúrgico se evaluaron por 15 días la temperatura de la zona anátomo-quirúrgica, el edema, el color del área umbilical y la dehiscencia de la herida, mientras que la recidiva de la hernia, la fistulación y el apetito de los animales se evaluaron por dos meses. Para la medición de la temperatura se empleó termómetro infrarrojo digital marca Noncontact IR (Guangzhou Kyunion Co.Ltd.).

\section{RESULTADOS}

Temperatura del local quirúrgico. De la evaluación de la temperatura de la zona anátomo-quirúrgica se obtuvo un promedio de $37.5^{\circ} \mathrm{C}$ en todos los pacientes. Al interpretar la Figura 4, se puede decir que hacia el cuarto día el promedio de la temperatura alcanzó su máximo $\left(38.2^{\circ} \mathrm{C}\right)$ con tendencia a la normalización en los días siguientes.

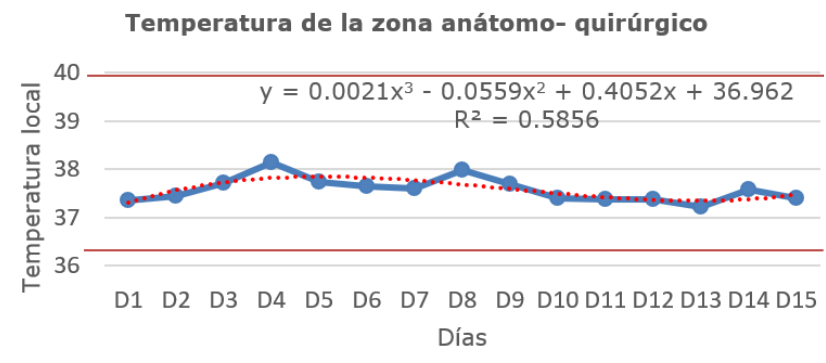

Figura 4. Representación gráfica de la línea de tendencia de la temperatura cutánea en la zona intervenida quirúrgicamente, evaluada clínicamente durante los 15 días de post-operatorio. 
La tendencia de la temperatura siguió un comportamiento polinómico de orden 3 en relación al tiempo, explicado por la siguiente ecuación lineal:

$y=0.0021 x^{3}-0.0559 x 2+0.4052 x+36.962$ $\left(R^{2}=0.5856\right)$

Donde:

$y=$ Temperatura de la zona anátomo-quirúrgica $\mathrm{X}=$ Unidades en días

El coeficiente de determinación indicó que este comportamiento está explicado en un $58 \%$ por la ecuación lineal anterior.

Edema. Los animales mostraron una disminución significativa de la inflamación que generó la manipulación tisular e implantación del PVC, con halos tumorales de un $\mathrm{cm}$ a las 24 horas post-cirugía y en algunos pacientes sin presencia del mismo, quedando en clasificación grado 1 y 0 respectivamente. Además; se puede inferir que la tendencia del edema fue a involucionar, alcanzando completa normalidad al final de la evaluación (Figura 5).

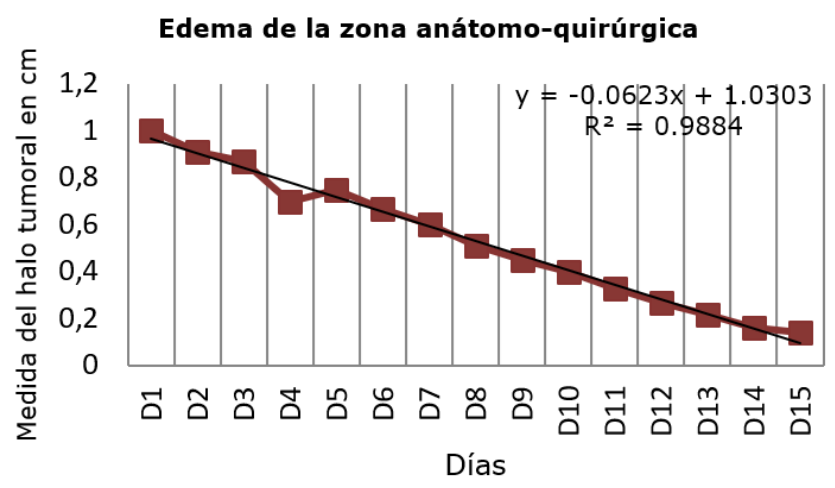

Figura 5. Línea de tendencia del halo tumoral durante 15 días de evaluación post-quirúrgica.

La tendencia del halo tumoral siguió un comportamiento lineal decreciente en función del tiempo, explicado por la siguiente ecuación lineal:

$y=-0.0623 x+1.0303\left(R^{2}=0.9884\right)$

Donde:

$y=$ Medida del halo tumoral en centímetros

$\mathrm{x}=$ Unidades en días
El coeficiente de determinación indicó que este comportamiento está explicado en un $98 \%$ por la ecuación lineal anterior.

Color del local quirúrgico. Se observó que el $100 \%$ de los animales en los primeros siete días post-quirúrgico presentaron un enrojecimiento de la zona de la herida ubicándose dentro de la clasificación 1 y a partir del octavo día hasta el final de la evaluación la tendencia fue a la normalidad, clasificándose como grado 0 (Figura 6).

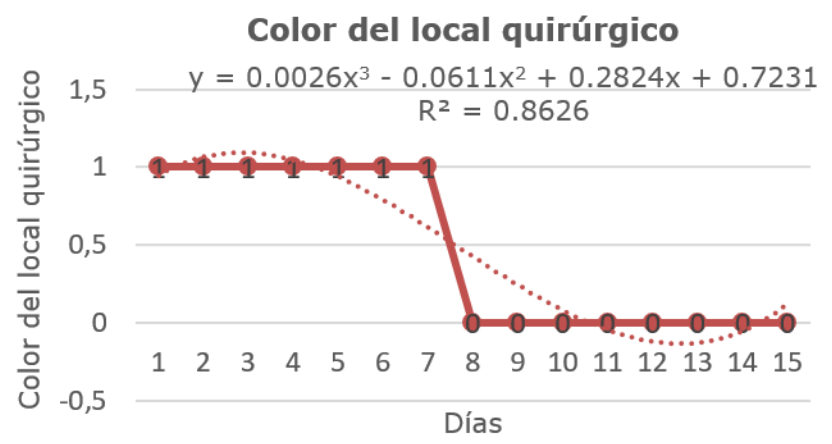

Figura 6. Línea de tendencia del color en el local quirúrgico, evaluado clínicamente durante 15 días de post-operatorio.

La tendencia del color siguió un comportamiento polinómico de orden 3 en función del tiempo, explicado por la siguiente ecuación lineal:

$y=0.0026 x^{3}-0.0611 x 2+0.2824 x+0.7231$ $\left(R^{2}=0.8626\right)$

Donde:

$y=$ Color del local quirúrgico ( 1 enrojecido y 0 normal)

x= Unidades en días

El coeficiente de determinación está explicado en un $86 \%$ por la ecuación lineal anterior.

Temperatura del local quirúrgico. En los 15 días de post-operatorio se obtuvo un promedio de $37.5^{\circ} \mathrm{C}$ en todos los pacientes; indicando que el $100 \%$ de los animales tuvieron un comportamiento dentro de la normalidad fisiológica. Al cuarto día el promedio de la temperatura alcanzó su máximo $\left(38.2^{\circ} \mathrm{C}\right)$ con tendencia a la normalización en los días siguientes (Figura 7). 


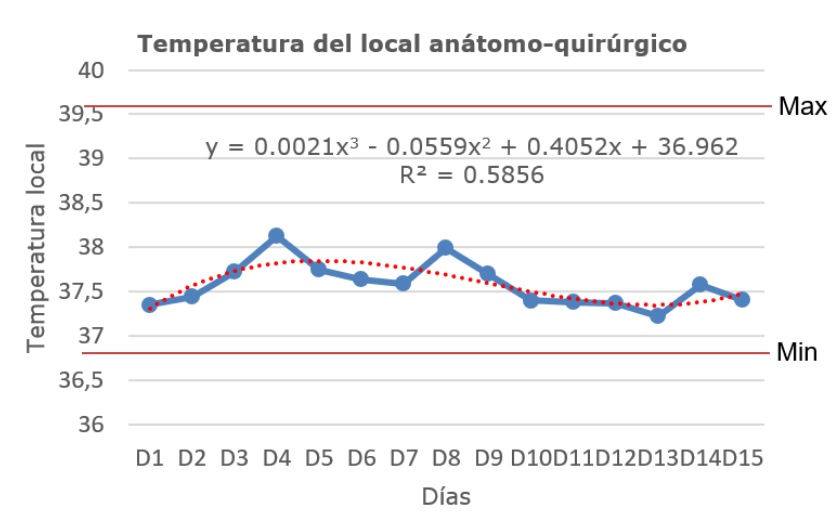

Figura 7. Línea de tendencia de la temperatura cutánea en la zona intervenida quirúrgicamente, evaluada clínicamente durante los 15 días de post-operatorio.

La tendencia de la temperatura cutánea de la zona intervenida quirúrgicamente siguió un comportamiento polinómico de orden 3 en función del tiempo, explicado por la siguiente ecuación lineal:

$y=0.0021 x^{3}-0.0559 x 2+0.4052 x+36.962$ $\left(R^{2}=0.5856\right)$.

Donde:

$y=$ Temperatura de la zona quirúrgica

$\mathrm{x}=$ Unidades en días

El coeficiente de determinación indicó que este comportamiento de la temperatura cutánea de la zona intervenida quirúrgicamente en el período del tiempo evaluado, esta explicado en un $58 \%$ por la ecuación lineal anterior

Dehiscencia de la herida. Al evaluar el proceso de cierre y cicatrización de la herida el $100 \%$ de los animales evolucionaron favorablemente quedando dentro de la clasificación 0 (ausente) de acuerdo a la clasificación de Martínez et al (3).

Recidiva de la hernia. Del total de los animales evaluados se encontró que 14 ( $87.5 \%)$ no presentaron recidiva de la hernia quedando en la clasificación 0 y dos $(12.5 \%)$ presentaron recidiva ubicados en la clasificación 1 de acuerdo con la clasificación de Martínez et al (3).

Fistulación. El $100 \%$ de los animales no mostraron signos clínicos compatibles con la condición, teniendo evolución favorable del cierre de la herida y del proceso cicatrizal.
Apetito y ganancia de peso. El $100 \%$ de los animales mostraron comportamiento normal del apetito, quedando en la clasificación 1. Esta variable se vio reflejada en la ganancia de peso de los cerdos que fue de $0.686 \mathrm{~kg} /$ día, calculada mediante la siguiente ecuación $(7,8)$ :

\section{GMDP $=(($ PESO FINAL-PESO INICIAL ) $) /($ DÍAS )}

La tendencia de la ganancia de peso postquirúrgico (Figura 8), siguió un comportamiento exponencial en relación al tiempo, como lo demuestra la siguiente ecuación lineal:

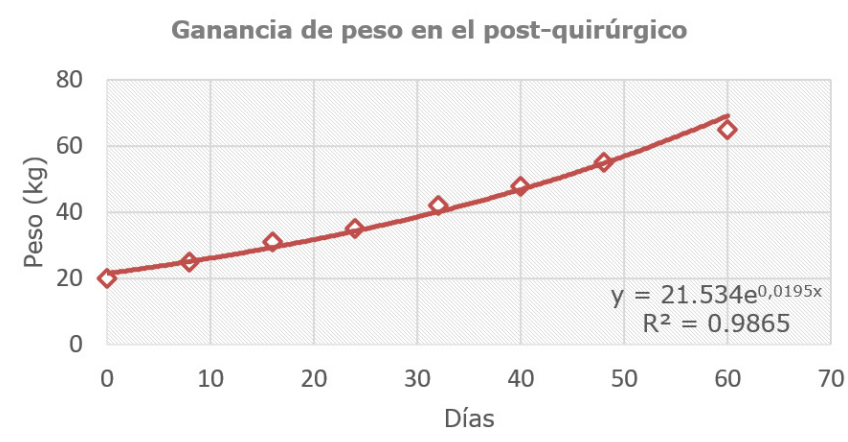

Figura 8. Línea de tendencia de la ganancia de peso diaria en el post-quirúrgico.

$y=21.534 e^{0,0195 x}\left(R^{2}=0.9865\right)$

Donde:

$\mathrm{y}=$ Peso en $\mathrm{kg}$

$\mathrm{X}=$ Unidades en días

El coeficiente de determinación indicó que este está explicado en un $98 \%$ por la ecuación exponencial anterior.

\section{DISCUSIÓN}

Evaluación post-quirúrgica. Los animales intervenidos quirúrgicamente con implantación del PVC para la corrección de hernias umbilicales no presentaron complicaciones post-operatorias como edema y rubor crónico. Los resultados son similares a los reportados con el empleo de la técnica de Mayo, con implantación de fascia abdominal autógena y con empleo del compuesto de látex, poliamida y polilisina al $0.1 \%(2,3,9)$.

La temperatura del local anátomo-quirúrgico, tuvo un comportamiento dentro de la normalidad fisiológica. Además; se podría inferir que el 
material implantado entre el peritoneo parietal y los músculos abdominales no provocó infección ni reacción inflamatoria más allá de los límites fisiológicos como los reportados en estudios de casos con implantes de mallas para corregir hernias umbilicales en humanos donde la infección complicante está entre $0.13-0.58$ con IC $=95 \%(10,11)$.

Edema. El proceso inflamatorio se dio dentro de condiciones fisiológicas, porque el primer día de evaluación reveló aumento máximo de un $\mathrm{cm}$, involucionando con el tiempo hasta la normalidad al día 15. Los resultados son semejantes a los de otros autores, los cuales afirman que la fase inflamatoria tiene su inicio a los 16 minutos y presenta duración de hasta seis días $(12,13,14)$. Este signo cardinal de la inflamación se debe a la respuesta vascular y a la respuesta celular que son estimuladas por quimiocinas como: la histamina tipo I, factores derivados del complemento ( $\mathrm{C} 3 a$ y $\mathrm{C} 5 \mathrm{a}$ ), prostaglandinas y óxido nitroso, que aumenta la permeabilidad capilar para la diapédesis con la consiguiente formación del edema $(12,13,14)$.

Dehiscencia de la herida. No se presentó, difiriendo de resultados de otros trabajos que utilizan auto-injerto de fascia abdominal bovina y malla sintética que reportan dehiscencia de la herida en un 30 y $60 \%$ en los pacientes evaluados, siendo la constante el edema intenso de la piel y en el tejido sub-cutáneo $(3,14)$.

Recidiva de la hernia. Dos animales (12.5\%) presentaron recidiva, que pudo deberse a que los tejidos con procesos inflamatorios activos tienen actividad colagenasa con capacidad para desdoblar al colágeno e influir negativamente en su síntesis, y que se refleja en menor resistencia del tejido a las tensiones $(11,12,13)$, como ocurre con el contenido abdominal sobre el local quirúrgico pudiendo favorecer la aparición de recidiva. Este porcentaje de recidiva fue semejante al reporte del $10.4 \%$ en correcciones de eventraciones con la técnica de Rives-Stoppa en humanos (14). Además; cabe anotar que el comportamiento de los cerdos a resistirse a la hora de las curaciones sumadas a que los animales del estudio no tuvieron restricción alimenticia, pudieron ser variables que influyeron para la recidiva. Los resultados del estudio difieren con otro reporte que no tuvo complicación (3) y coincide con reportes del 5 al $10 \%$ de recidiva en herniorrafias con mallas protésicas en humanos (6).
En este estudio la implantación del PVC como material protésico para herniorrafias, mostró una efectividad del $87.5 \%$. Hay que decir que los animales que recidivaron corresponden a los de mayor temperamento nervioso y se encontró el material roto en los puntos de sutura. El desgarro del PVC se pudo confirmar post-descarte de los animales. Los resultados están en concordancia con otros estudios que reportan ruptura del material en un $67 \%$ y $83 \%$ cuando se empleó como prótesis en el manejo de abdomen abierto en humanos (4).

Fistulación. Aunque no se realizaron pruebas que evaluaran la biocompatibilidad del PVC y de la poliamida utilizada para suturar y fijar el material protésico a la fascia abdominal, se presume que fueron aceptados por el tejido receptor porque no se evidenciaron clínicamente procesos fistulosos o formación de granulomas, coincidiendo con otros reportes $(6,15)$.

Ganancia de peso. El consumo de alimento de los animales fue normal con ganancia media de peso diario de 686 gr/día (Figura 8), lo que permite presumir que la cirugía correctiva con bolsa de PVC, no afecta el comportamiento de los cerdos, porque sus parámetros de conversión alimenticia se dieron entre el rango de los ya establecidos en el manejo de ésta especie animal que es de $659 \pm 193 \mathrm{gr} /$ día (16).

En conclusión, los resultados del presente estudio se pueden concluir que la herniorrafia en cerdos con la técnica de Rives-Stoppa modificada utilizando PVC como malla, fue efectiva; con ventajas como baja recidiva, nula presencia de fístula, sin adherencias o granulomas, corta convalecencia de los animales (de siete a 10 días), bajo costo del material y su fácil consecución. Los pacientes intervenidos mantuvieron el apetito, lo que garantizó la ganancia de peso acorde con la fase productiva. Por todo lo anterior, se recomienda su uso en los programas de producción porcina.

\section{Conflicto de intereses}

Los autores declaran que no tienen ningún conflicto de intereses en relación con el trabajo presentado.

\section{Agradecimiento}

Los autores agradecen a la Facultad de Medicina Veterinaria y Zootecnia de la Universidad de Córdoba, Colombia por el apoyo logístico y económico. 


\section{REFERENCIAS}

1. Flecknell P. Anestesia de animales de laboratorio: introducción practica para investigadores y técnicos. 2 Ed. País de publicación; Academic Press Ltd; 1998. https://www.editorialacribia.com/libro/ anestesia-de-animales-de-laboratoriointroduccion-practica-para-investigadoresy-tecnicos 53659/

2. Reyes I, Pérez E, Pérez $F$ y Reyes L. Malformaciones congénitas en la crianza de cerdos del sector no especializado en el oriente de cuba. Arch Zootec. 2010; 59(228):601-604. http://scielo.isciii.es/pdf/ azoo/v59n228/art14.pdf

3. Martínez M, Oviedo C, Ballut J. Evaluación clínica de hernioplastia umbilical en bovinos: empleo de fascia abdominal autógena. Rev MVZ Córdoba 2010; 15(2):2111-2117. https://doi.org/10.21897/rmvz.322 _

4. Borraez, O. Abdomen Abierto: Utilización del Polivinilo. Rev Colomb Cir 2008; 23(4):204-209. https://encolombia.com/ medicina/revistas-medicas/cirugia/vc-161/ cirugia16101-abdomen/

5. Ochoa G, Marino C, Riveros S, Morales $E$, Jarry $C$, et al. Implementación de un Modelo Porcino de Trasplante Hepático en Chile. Rev Cirugía. 2020; 72(5). https:// doi.org/10.35687/s2452-45492020005704

6. Acevedo A, Justiniano J, Lombardi J. técnica de Rives-Stoppa en las eventraciones de la línea media: prescindencia de la fijación perimetral de la malla. Rev Chil Cir 2014; 66(2):146-152. http://dx.doi.org/10.4067/ $\underline{\text { S0718-40262014000200009 }}$ -

7. Hernández J, Herrera M, Rodero E, Vargas S, Villarreal O, Reséndiz R, Carreón L. y Sierra A. Tendencia en el crecimiento de cabritos criollos en sistemas extensivos. Arch Zootec. 2005; 54:429-436. http://www.redalyc.org/ articulo.oa?id $=49520748$

8. Rabelo R, Ferreira F, Paulo N, Flávia A, Franco L, Oliveira $\mathrm{M}$ et al. Emprego do compósito látex, poliamida e polilisina a $0.1 \%$ na correção cirúrgica de hérnias umbilicais recidivantes em bovinos leiteiros. Acta Sci Vet 2005; 33(2):169-175. https://doi.org/10.22456/1679-9216.14796 .
9. Senet P. Fisiología de la cicatrización cutánea. EM-consulte. 2008. https://www.emconsulte.com/es/article/119845/resume/ fisiologia-de-la-cicatrizacion-cutanea

10. Lora A, Vega N, Barrios P, Ruiz P. Hernia umbilical: un problema no resuelto. Iatreia 2019; 32(4):288-297. https://doi. org/10.17533/udea.iatreia. 28

11. Salgado A, Salgado A, Arriba L. Nuevas tendencias en regeneración tisular: fibrina rica en plaquetas y leucocitos. Rev Esp Cir Oral Maxilofac. 2017; 39(2):91-98. http:// dx.doi.org/10.1016/j.maxilo.2016.03.001

12. Vargas A. Regeneración y cicatrización. Rev Act Clin Med. 2014; (43):2256-2260. http:// www.revistasbolivianas.org.bo/pdf/raci/ v43/v43 a03.pdf

13. Guarín C, Quiroga P, Landínez N. Wound healing process of skin, endogenous fields related and chronic wounds. Rev Fac Med. 2013; 61(4):441-448. https://revistas. unal.edu.co/index.php/revfacmed/article/ view/42815/47623

14. Acevedo A, Justiniano J, Lombardi J. técnica de Rives-Stoppa en las eventraciones de la línea media. Prescindencia de la fijación perimetral de la malla. Rev Chil Cir 2014; 66(2):146-152. http://dx.doi.org/10.4067/ $\underline{\text { S0718-40262014000200009 }}$

15. López S, Pérez A, Martínez M. Técnica de Mastoby para preparar ovinos receladores. Rev Med Vet 2018; (36):15-26. http:// dx.doi.org/10.19052/mv.5168

16. Benítez A, Gómez A, Hernández J, Navarrete $\mathrm{R}$, Moreno $\mathrm{L}$. Evaluation of the productive and economic performance of feed for fattening pigs. Rev Abanico Veterinario 2015; 5(3):36-41. https://abanicoacademico. $\mathrm{mx} / \mathrm{revistasabanico/index.php/abanico-}$ veterinario/article/view/80. 